\title{
Quantum Chemical Calculations and Molecular Docking Studies of Some NSAID Drugs (Aceclofenac, Salicylic Acid, and Piroxicam) as 1PGE Inhibitors
}

\author{
S. Suresh, ${ }^{1,2}$ Sethu Gunasekaran, ${ }^{3}$ and Shanmugam Srinivasan ${ }^{2}$ \\ ${ }^{1}$ Department of Physics, Dr. M.G.R. Educational and Research Institute University, Chennai, India \\ ${ }^{2}$ Department of Physics, Presidency College, Chennai, India \\ ${ }^{3}$ Research and Development Center, St. Peter's University, Chennai, India \\ Correspondence should be addressed to S. Suresh; sureshthanjai2003@gmail.com
}

Received 6 May 2016; Accepted 10 August 2016

Academic Editor: Soni Mishra

Copyright ( 92016 S. Suresh et al. This is an open access article distributed under the Creative Commons Attribution License, which permits unrestricted use, distribution, and reproduction in any medium, provided the original work is properly cited.

\begin{abstract}
The molecular structure of the three compounds Aceclofenac (I), Salicylic Acid (II), and Piroxicam (III) has been determined using Gaussian 03W program with B3LYP method using 6-311++G $(\mathrm{d}, \mathrm{p})$ basis set calculations. The molecular structures were fully optimized with atomic numbering scheme adopted in the study. To understand the mode of binding and molecular interaction, the docking studies of compounds Aceclofenac (I), Salicylic Acid (II), and Piroxicam (III) have been carried out with prostaglandin H2 synthase-1 (1PGE) as target using induced fit docking. The molecular docking results show that the interactions and energy for Aceclofenac, Salicylic Acid, and Piroxicam show the best results when docked with prostaglandin H2 synthase-1 (1PGE). The hydrogen bonding interactions of compound I (Aceclofenac) are prominent with Arginine moiety, those of compound II (Salicylic Acid) are prominent with Tyrosine and Serine moieties, and compound III (Piroxicam) shows such interaction with Tyrosine and Arginine moieties. These interactions of prostaglandin H2 synthase-1 (1PGE) with substrates are responsible for governing COX-1 inhibitor potency which in turn is a direct measure of the potency of the drug.
\end{abstract}

\section{Introduction}

The nonsteroidal anti-inflammatory drugs (NSAIDs) have been among the most widely used therapeutic agents due to their anti-inflammatory, analgesic, and antipyretic effects [1]. Nonsteroidal anti-inflammatory drugs (NSAIDs) are the most frequently prescribed drugs worldwide in musculoskeletal disorders. The mode of action of NSAIDs is by blocking the cyclooxygenase (COX) enzyme and consequently the biosynthesis of prostaglandins [2]. Two isoforms of the COX enzyme have been characterized: cyclooxygenase1 (COX-1) and cyclooxygenase-2 (COX-2) [3]. Although both isoforms catalyze the same biochemical transformation, they are subject to a different expression regulation. COX-1 is a constitutive enzyme and is responsible for the physiological function of prostaglandins like maintenance of the integrity of the gastric mucosa and provides adequate vascular homeostasis, whereas COX-2 is an inducible enzyme and is expressed only after an inflammatory stimulus [4]. Action of NSAIDs differs due to their different selectivity for COX-2 and COX-1. An NSAID that blocks COX-2 but not COX-1 might reduce pain and inflammation in joints but leave the stomach lining alone [5]. Prostaglandin H2 synthase is a key enzyme in the biosynthesis of prostaglandins mediating inflammation and other important physiological processes. Prostaglandins and thromboxanes generated via the COX-1 and COX-2 pathways are identical molecules and therefore have identical biological effects. Under the influence of COX-1, prostaglandins maintain the integrity of the gastric mucosa, mediate normal platelet function, and regulate renal blood flow [6].

In the previous study, the compound structures of Aceclofenac (I), Salicylic Acid (II), and Piroxicam (III) obtained crystallographically have been compared with their optimized structures obtained by B3LYP method using 6-311++G $(\mathrm{d}, \mathrm{p})$ basis set [7-9]. To the best of our knowledge, molecular 


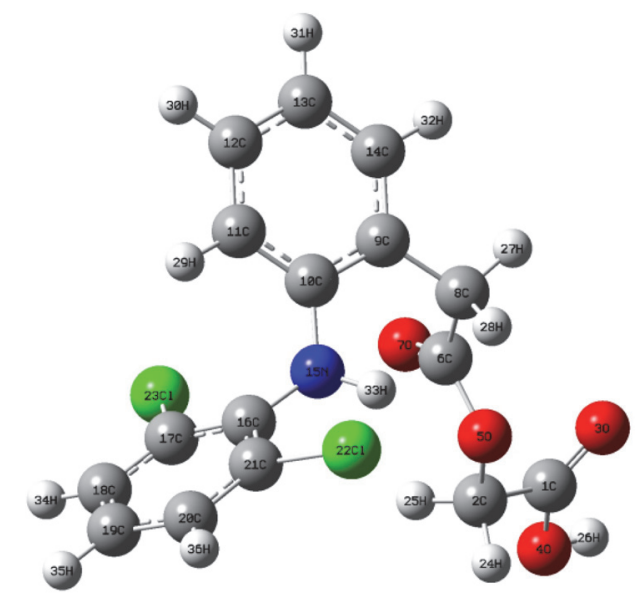

FIGURE 1: Optimized structure of compound I (Aceclofenac) with atom number.

docking, molecular modelling, and stability studies have not been reported so far for the compounds Aceclofenac (I), Salicylic Acid (II), and Piroxicam (III). In this study, molecular modelling and induced fit docking studies have been carried out for the above compounds with prostaglandin $\mathrm{H} 2$ synthase-1 (PDB ID: 1PGE) as target to understand the mode of binding. A higher level of binding with prostaglandin synthase usually indicates a strong anti-inflammatory action. The total energy, nuclear repulsion energy, total dipole moment, and polarizability of all the three compounds have been obtained from quantum chemical calculations, which is clearly shown the compounds I, II, and III molecular properties.

\section{Quantum Chemical Calculations}

Quantum chemical calculations have been performed at density functional theory with B3LYP method using 6$311++\mathrm{G}(\mathrm{d}, \mathrm{p})$ basis set using the Gaussian 03W program [10]. The molecular structures of compounds Aceclofenac (I), Salicylic Acid (II), and Piroxicam (III) in the ground state are optimized by density functional theory with B3LYP method using 6-311++G $(\mathrm{d}, \mathrm{p})$ basis set calculations.

Molecular docking studies of compounds Aceclofenac (I), Salicylic Acid (II), and Piroxicam (III) with the target protein were performed using Schrödinger software package [11]. The initial structure of the protein was taken from research collaborator for structural bioinformatics (RCSB) protein data bank (PDB) with code ID: 1PGE (http://www.wwpdb.org/). The imported protein structure was prepared by protein preparation wizard, the needed numbers of hydrogen atoms were added, and the refinement of the structure was carried out. The water molecules present in the crystallographic complex were deleted and chains $\mathrm{A}$ and $\mathrm{B}$ were removed in the crystal structure. This was followed by minimizing of the structures to a RMSD of $0.45 \AA$. Ligands were prepared using Ligprep module of the Maestro window of the Schrodinger Suite. The three ligands were subjected to full optimized minimization in the gaseous phase with density functional theory. Ligprep produces a number of structures from each input structure with various ionization states, tautomers, stereo chemistries, and ring conformations. Prepared ligand and receptor were used as the initial coordinates for docking purposes. In virtual docking studies, ligands are docked into active sites of the receptor, where the receptor is held rigid and the ligand is free to move. However, the assumption of rigid receptor can give misleading results, since in reality many proteins undergo side chain or backbone movements, or both, upon ligand binding. These changes allow the receptor to alter its binding site so that it more closely conforms to the shape and binding mode of the ligand [12].

\section{Results and Discussion}

3.1. Density Functional Theory Analysis. The optimized molecular geometries of the three compounds (I, II, and III) with atomic numbering schemes were obtained from density functional theory with B3LYP method using 6$311++\mathrm{G}(\mathrm{d}, \mathrm{p})$ basis set. The optimized molecular structures of the three compounds I, II, and III (Aceclofenac, Salicylic Acid, and Piroxicam) are shown in Figures 1-3, respectively. The molecular geometric parameters such as bond lengths, bond angles, and dihedral angles of compounds I, II, and III of the molecular structure conformation were obtained by crystallographic studies and compared with density functional theory with B3LYP method using 6-311++G $(\mathrm{d}, \mathrm{p})$ basis set calculations $[8,9]$. The three compounds I, II, and III (Aceclofenac, Salicylic Acid, and Piroxicam) are nonplanar molecular structures.

The molecular properties of the three compounds (I, II, and III), total energy, the nuclear repulsion energy, total dipole moment and polarizability components, obtained from quantum chemical calculation, are shown in Table 1.

\section{Molecular Docking (Induced Fit Docking)}

The three compounds with the prepared protein were performed using induced fit docking protocol of GLIDE version 5.6 from Schrödinger Suite, which is based on the GLIDE and Prime Refinement module. In induced fit docking, the 


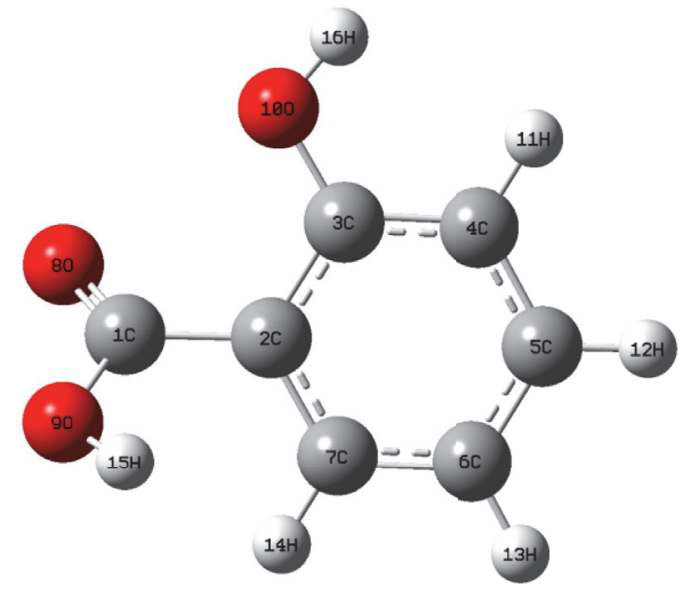

FIgURE 2: Optimized structure of compound II (Salicylic Acid) with atom number.

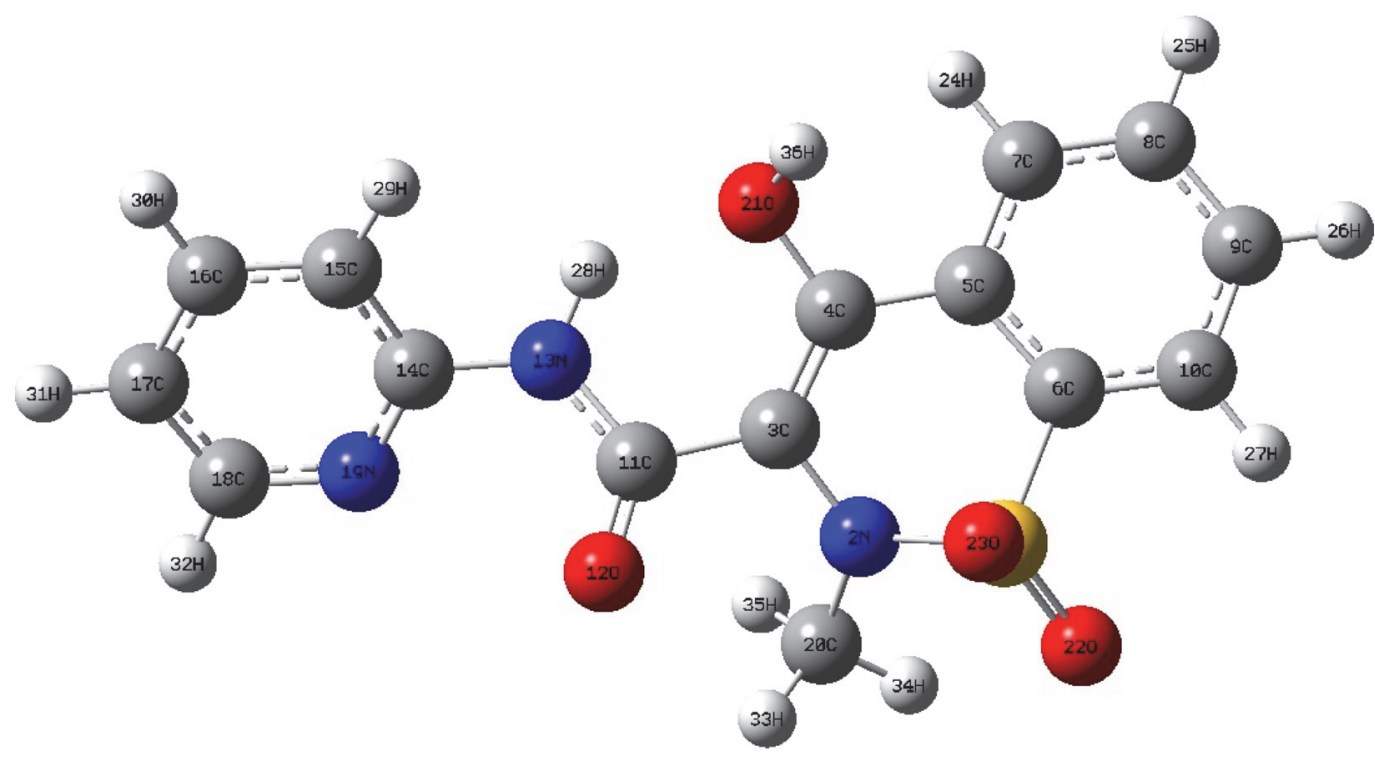

FIgURE 3: Optimized structure of compound III (Piroxicam) with atom number.

TABLE 1: Polarizability and nuclear repulsion energy of compounds I, II, and III.

\begin{tabular}{lccc}
\hline Parameters & Compound I & Compound II & Compound III \\
\hline Nuclear repulsion energy (Hartree) & 2181.8354 & 499.3075 & 2079.5662 \\
Polarizability & & & 350.0084 \\
XX & 242.6009 & 113.8430 & 244.1901 \\
YY & 254.9348 & 101.6600 & 142.8490 \\
ZZ & 208.4840 & 58.1443 & 8.0620 \\
Dipole moment (Debye) & 3.3180 & 5.7783 & -1442.9920 \\
Total energy (Hartree) & -1893.9236 & -496.1829 & \\
\hline
\end{tabular}

ligand and the receptor are flexible, which enables docking the ligand at the receptor's binding site. The structural conformation of the receptor to fit the ligand pose and ranking is done by Glide score (G-score) to find the best structure of the docked complex. Glide score is an empirical scoring function that approximates the ligand binding free energy. The PyMOL molecular graphics system was used to analyze the hydrogen bond interaction and preparation of high resolution images $[13,14]$.

The structure of prostaglandin $\mathrm{H} 2$ synthase-1 (1PGE) receptor shows that it may undergo backbone movement. Figure 4 represents the active site of the 3D structure of a target receptor protein molecule. It shows the side chains involved in the active site. So the single bonded flexible molecular 


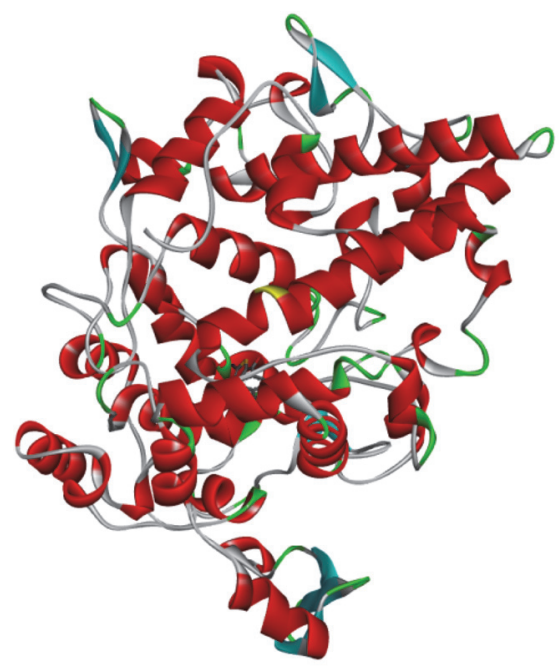

FIGURE 4: The Ribbon view of the crystal structure of prostogladin H2 synthase (1PGE).

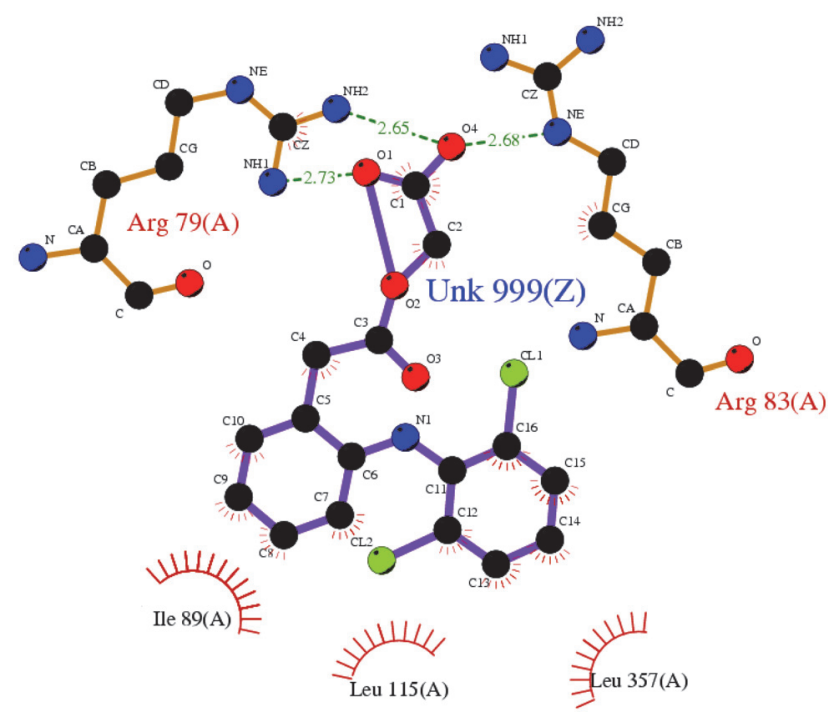

FIGURE 5: Graphical representation of interaction of ligand compound I at active binding site of 1PGE.

fragments are susceptible to conformational changes. These changes may permit alterations in the receptor. These changes may permit alterations in the receptor, so that it almost closely conforms to the shape of the ligands. Thus, the flexibility of receptor has been taken by induced fit docking in the Schrodinger Suite software [15].

The induced fit docking of compounds I, II, and III has been carried out with prostaglandin $\mathrm{H} 2$ synthase (1PGE) protein as target. The results of the induced fit docking Glide score and binding energy of selective COX-1 inhibitors are shown in Table 2, which displays the best docked poses. Using Schrodinger software, the binding Glide energy of Piroxicam with the target was calculated to be $-49.35 \mathrm{kcal} / \mathrm{mol}$ which was the best among the three compounds in the studies. This is followed by Aceclofenac which shows a binding Glide energy of $-41.35 \mathrm{kcal} / \mathrm{mol}$. Salicylic Acid shows the least binding Glide energy of the three at $-25.99 \mathrm{kcal} / \mathrm{mol}$.
TABLE 2: Glide score and binding Glide energy of selective COX-1 inhibitors 1PGE.

\begin{tabular}{lccc}
\hline S. number & Ligand & Glide score & $\begin{array}{c}\text { Glide energy } \\
(\mathrm{kcal} / \mathrm{mol})\end{array}$ \\
\hline 1 & Aceclofenac & -10.0773 & -41.3458 \\
2 & Salicylic Acid & -5.6022 & -25.9929 \\
3 & Piroxicam & -5.8742 & -49.3469 \\
\hline
\end{tabular}

Figures 5-7 depict the interactions of compounds I, II, and III with 1PGE.

The Glide scores are negative; higher negative values indicate more binding interactions. The green dotted line indicates $\mathrm{H}$-bond interactions with receptor. Table 3 shows the interactions of $\mathrm{H}$-bonding with receptors and ligands. It is observed that in compound I (Aceclofenac) the two 


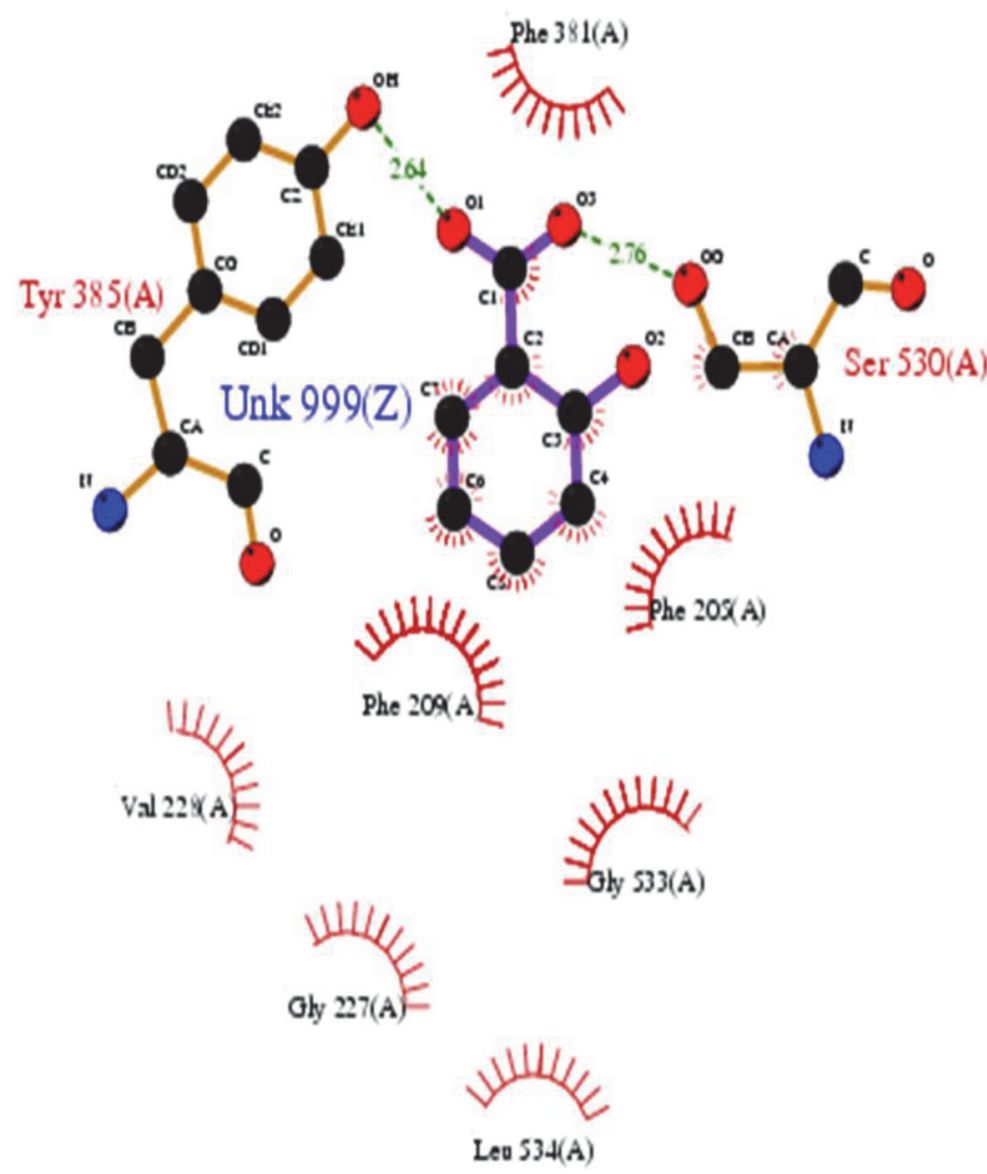

FIGURE 6: Graphical representation of interaction of ligand compound II at active binding site of 1PGE.

TABLE 3: Molecular interaction analysis of compounds I, II, and III docking hits.

\begin{tabular}{|c|c|c|c|c|}
\hline S. number & Compound name & Interacting atom (protein/cofactor) & Interacting atom (ligand) & Interaction distance $(\AA)$ \\
\hline \multirow{3}{*}{1} & \multirow{3}{*}{ Aceclofenac } & \multirow{2}{*}{ Arg79 } & $\mathrm{N}-\mathrm{H} \cdots \mathrm{O}$ & 2.65 \\
\hline & & & $\mathrm{N}-\mathrm{H} \cdots \mathrm{O}$ & 2.73 \\
\hline & & Arg83 & $\mathrm{N}-\mathrm{H} \cdots \mathrm{O}$ & 2.68 \\
\hline \multirow{2}{*}{2} & \multirow{2}{*}{ Salicylic Acid } & Tyr385 & $\mathrm{O}-\mathrm{H} \cdots \mathrm{O}$ & 2.64 \\
\hline & & Ser530 & $\mathrm{O}-\mathrm{H} \cdots \mathrm{O}$ & 2.76 \\
\hline \multirow{4}{*}{3} & \multirow{4}{*}{ Piroxicam } & \multirow{2}{*}{ Arg83 } & $\mathrm{N}-\mathrm{H} \cdots \mathrm{O}$ & 2.79 \\
\hline & & & $\mathrm{N}-\mathrm{H} \cdots \mathrm{O}$ & 3.22 \\
\hline & & Isf800 & $\mathrm{O}-\mathrm{H} \cdots \mathrm{O}$ & 3.33 \\
\hline & & Tyr355 & $\mathrm{O}-\mathrm{H} \cdots \mathrm{O}$ & 3.16 \\
\hline
\end{tabular}

hydroxyl groups interact with nitrogen atom of amide group of Arginine residues Arg79 and Arg83 at a distance of $2.65 \AA$, $2.73 \AA$, and $2.68 \AA$, respectively. In compound II (Salicylic Acid), the hydroxyl group interacts with oxygen atom of amide group of the Tyrosine and Serine residues Tyr385 and Ser530 at a distance of $2.64 \AA$ and $2.76 \AA$. In compound III (Piroxicam), the carboxylic group interacts with $\mathrm{N}$ atoms of the amide group of residue Arg83.

It also interacts with the hydroxyl groups with oxygen atom of amide group of Tyrosine (Tyr355) and Isf 800 residues. The oxygen atom of carboxylic group of the crystal ligand interacts with amide group of Arginine residue (Arg83) at a distance of $2.79 \AA$ and $3.22 \AA$. Moreover, the hydroxyl groups interact with oxygen atom of amide group of Tyrosine (Tyr355) and Isf 800 residues at a distance of $3.13 \AA$ and $3.33 \AA$, respectively. The docking results obtained the Glide energy of the three compounds Aceclofenac (I), Salicylic Acid (II), and Piroxicam (III) as -10.077335, -5.602205, and -5.874264 , respectively. In general, it can be stated that all the three compounds, namely I, II, and III, exhibit 


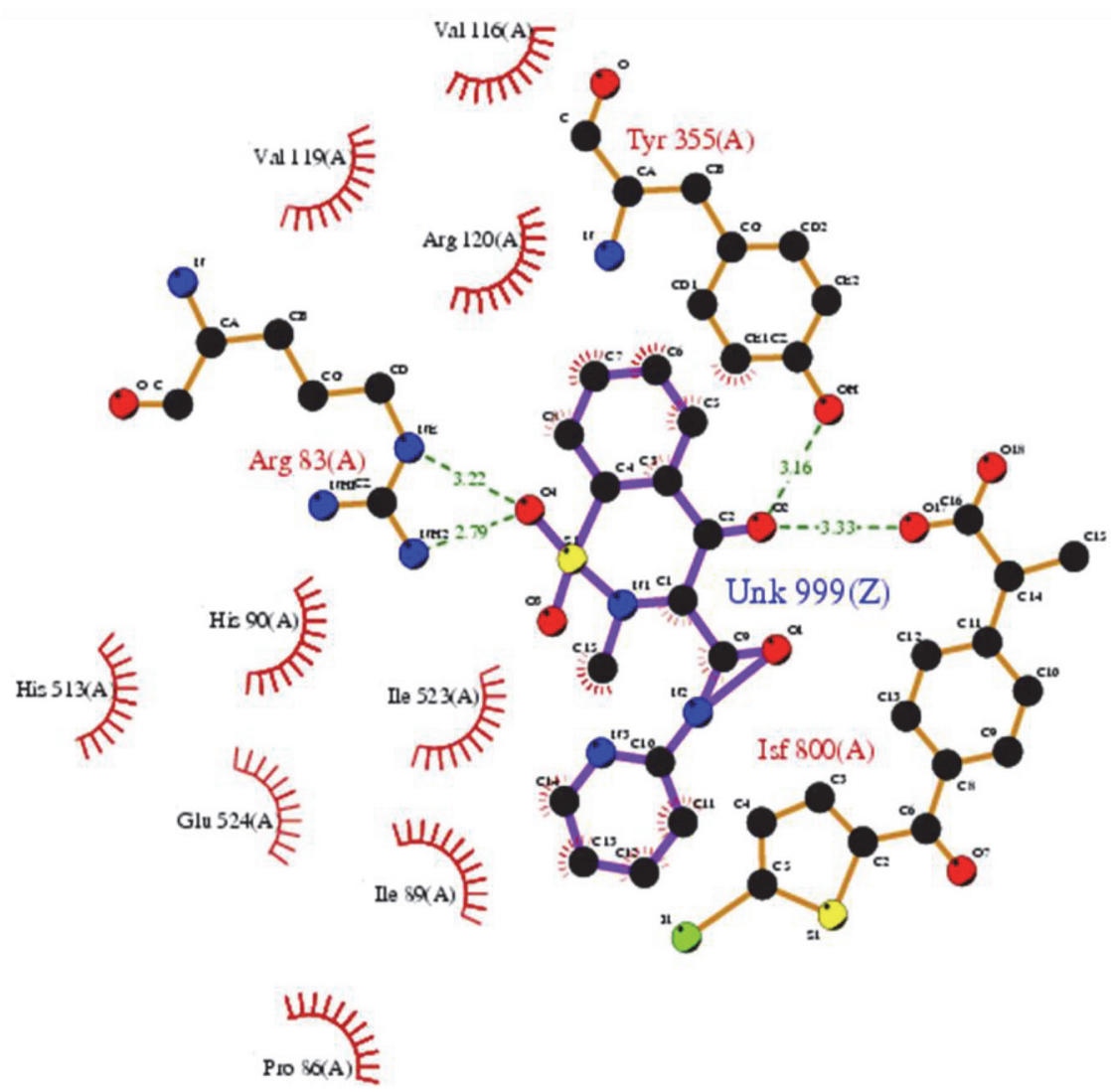

FIGURE 7: Graphical representation of interaction of ligand compound III at active binding site of 1PGE.

considerable binding tendencies. However, compound III supercedes the others in this respect. The stabilization of protein-ligand complex is mainly due to the H-bonds and hydrophobic interactions, which results in the inhibition of the COX-1 activity.

\section{Conclusion}

In this paper, we have reported that the docking studies of compounds Aceclofenac (I), Salicylic Acid (II), and Piroxicam (III) have been carried out with prostaglandin $\mathrm{H} 2$ synthase-1 (1PGE) as target using induced fit docking. The docking results show that the hydrogen bonds, hydrophobic interactions, and Glide energy of the three compounds Aceclofenac (I), Salicylic Acid (II), and Piroxicam (III) show the best binding capabilities when docked with prostaglandin $\mathrm{H} 2$ synthase (1PGE). In the comparison of the three compounds, the Glide energy of Piroxicam is $-49.35 \mathrm{kcal} / \mathrm{mol}$ with the target being the best binding affinity among the three compounds. The three compounds I, II, and III showed best docking score, Glide energy, hydrogen bonds, and hydrophobic interactions to stabilize the protein-ligand complex.

\section{Competing Interests}

The authors declare that they have no competing interests.

\section{References}

[1] J. R. Vane, "Inhibition of prostaglandin synthesis as a mechanism of action for aspirin-like drugs," Nature: New biology, vol. 231, no. 25, pp. 232-235, 1971.

[2] M. K. O’Banion, H. B. Sadowski, V. Winn, and D. A. Young, "A serum- and glucocorticoid-regulated 4-kilobase mRNA encodes a cyclooxygenase-related protein," The Journal of Biological Chemistry, vol. 266, no. 34, pp. 23261-23267, 1991.

[3] A. Palomer, F. Cabré, J. Pascual et al., "Identification of novel cyclooxygenase-2 selective inhibitors using pharmacophore models," Journal of Medicinal Chemistry, vol. 45, no. 7, pp. 14021411, 2002.

[4] S. Sarat, Non-Steroidal Anti Inflammatory Drugs an Overview, Pharma Med Press India, 2008.

[5] L. J. Crofford, "COX-1 and COX-2 tissue expression: implications and predictions," The Journal of Rheumatology, vol. 24, supplement 49, pp. 5-9, 1997.

[6] S. Suresh, S. Gunasekaran, and S. Srinivasan, "Studies of the molecular geometry, vibrational spectra, frontier molecular orbital, nonlinear optical and thermodynamics properties of Aceclofenac by quantum chemical calculations," Spectrochimica Acta-Part A: Molecular and Biomolecular Spectroscopy, vol. 125, pp. 239-251, 2014.

[7] S. Suresh, S. Gunasekaran, and S. Srinivasan, "Spectroscopic (FT-IR, FT-Raman, NMR and UV-Visible) and quantum chemical studies of molecular geometry, Frontier molecular orbital, 
NLO, NBO and thermodynamic properties of salicylic acid," Spectrochimica Acta Part A: Molecular and Biomolecular Spectroscopy, vol. 132, pp. 130-141, 2014.

[8] S. Suresh, S. Gunasekaran, and S. Srinivasan, "Vibrational spectra (FT-IR, FT-Raman), frontier molecular orbital, first hyperpolarizability, NBO analysis and thermodynamics properties of piroxicam by HF and DFT methods," Spectrochimica Acta Part A: Molecular and Biomolecular Spectroscopy, vol. 138, pp. 447-459, 2015.

[9] Gaussian 03, Gaussian Inc, Pittsburgh, Pa, USA, 2003.

[10] Glide Version 5.6, Schrödinger, LLC, New York, NY, USA, 2010.

[11] http://www.wwpdb.org/.

[12] N. Moitessier, E. Therrien, and S. Hanessian, "A method for induced-fit docking, scoring, and ranking of flexible ligands. Application to peptidic and pseudopeptidic $\beta$-secretase (BACE 1) inhibitors," Journal of Medicinal Chemistry, vol. 49, no. 20, pp. 5885-5894, 2006.

[13] W. L. DeLano, The PyMol Molecular Graphic System, 2002.

[14] B. Q. Wei, L. H. Weaver, A. M. Ferrari, B. W. Matthews, and B. K. Schoichek, "Testing a flexible-receptor docking algorithm in a model binding site," Journal of Molecular Biology, vol. 337, no. 5, pp. 1161-1182, 2004.

[15] H. B. Broughton, "A method for including protein flexibility in protein-ligand docking: improving tools for database mining and virtual screening," Journal of Molecular Graphics and Modelling, vol. 18, no. 3, pp. 247-257, 2000. 

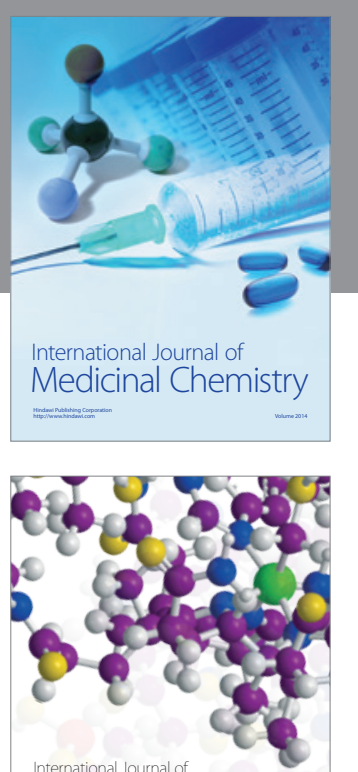

Carbohydrate Chemistry

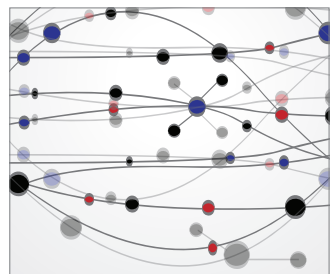

The Scientific World Journal
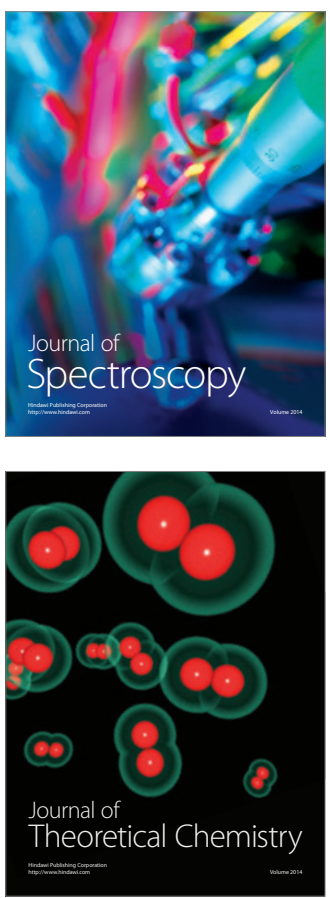
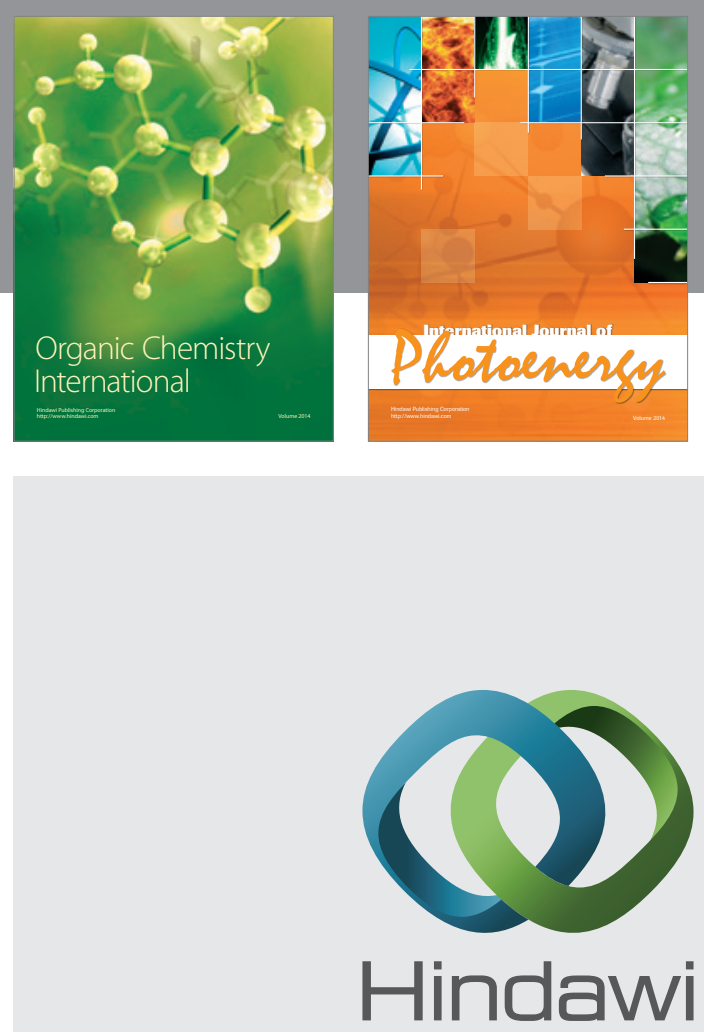

Submit your manuscripts at

http://www.hindawi.com

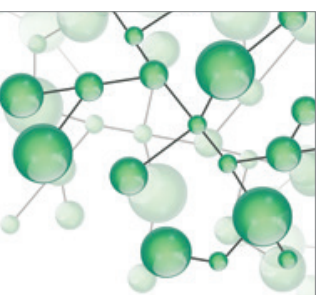

International Journal of

Inorganic Chemistry

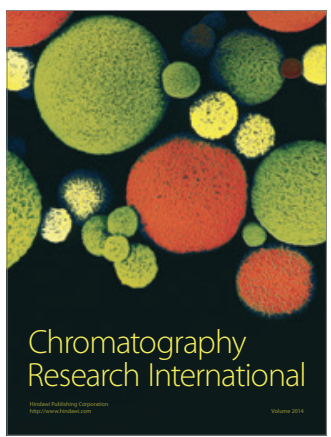

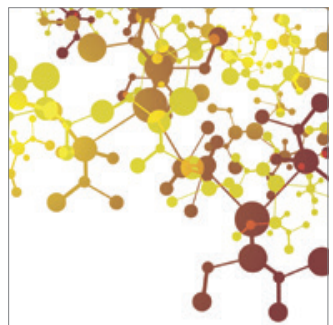

Applied Chemistry
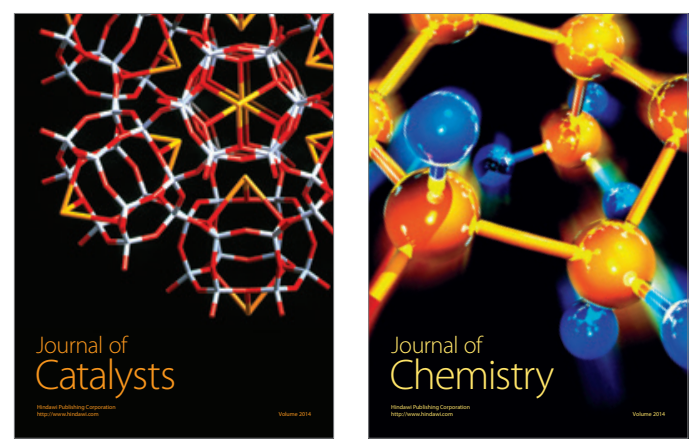
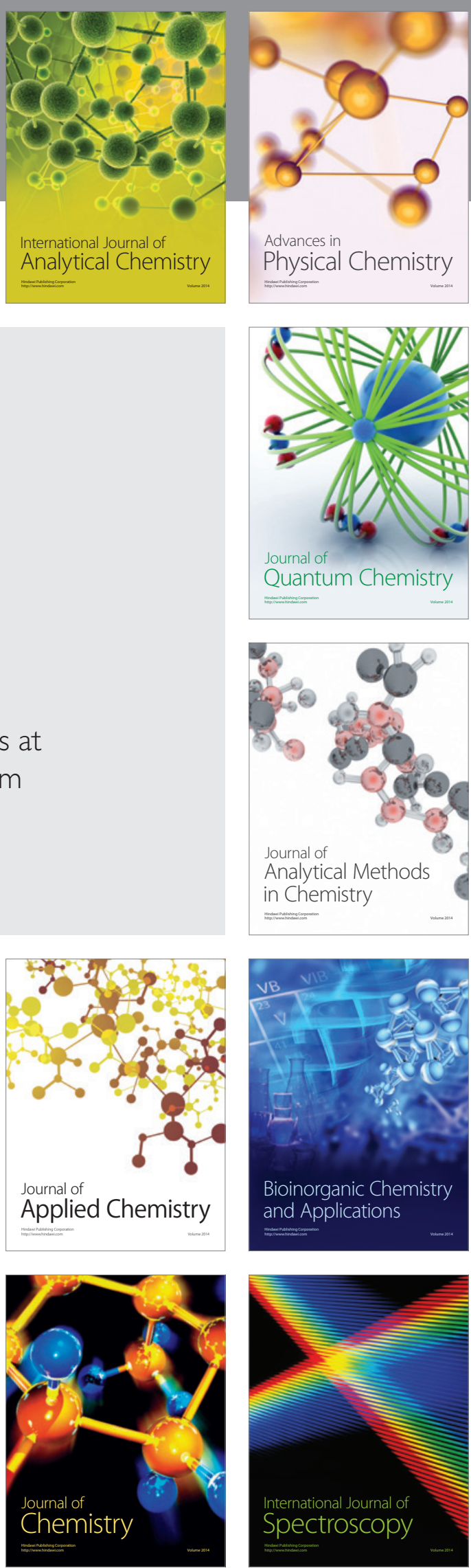